\title{
SUPERCLUSTERING OF DAMPED LYMAN-ALPHA ABSORPTION SYSTEMS AT REDSHIFTS ABOVE TWO
}

\author{
PAUL J. FRANCIS \\ Steward Observatory, University of Arizona, Tucson, Arizona 85721 \\ Electronic mail: pjf@as.arizona.edu \\ Paul C. Hewett \\ Institute of Astronomy, Madingley Road, Cambridge, CB3 OHA, England \\ Electronic mail: phewett@mail.ast.cam.ac.uk \\ Received 1992 November 24; revised 1992 December 21
}

\begin{abstract}
Observations of two high redshift quasi-stellar objects (QSOs) $(z=3.23$ and $z=3.18)$ separated by 8 arcmin on the sky are presented. Two strong Lyman- $\alpha$ absorption systems are seen in each QSO, and in both QSOs, one absorber is at a redshift of 2.380 and the other is at a redshift of 2.853 . The velocities of the absorption systems differ between the lines of sight by only $\sim 300 \mathrm{~km} \mathrm{~s}^{-1}$ in both cases. To cover both lines of sight, the absorbers would need a transverse comoving size of $\sim 6 h_{100}^{-1} \mathrm{Mpc}$ (for $q_{0}=0.5$ ). The neutral hydrogen column densities inferred from the profiles lie between $1.2 \times 10^{18}$ and $1.2 \times 10^{20} \mathrm{~cm}^{-2}$, and low ionization metal lines are associated with at least one of the absorption systems. The a posteriori joint probability of seeing these redshift coincidences is very low, $\sim 3 \times 10^{-4}$. If the absorbers are clustered, the probability of seeing the wavelength matches is enhanced, but a 20 Mpc scale supercluster with an absorber overdensity of $\sim 30$ is required to give even a 5\% probability of seeing the observed wavelength matches. If, alternatively, individual giant gas clouds cover both lines of sight, they must have masses in excess of $10^{11} \mathscr{M}_{\odot}$. Any evidence for $\sim 5 \mathrm{Mpc}$ structure at these redshifts poses challenge to bottom-up structure formation scenarios such as the standard Cold Dark Matter model.
\end{abstract}

\section{INTRODUCTION}

During the construction of the Large Bright QSO Survey (LBQS, Foltz et al. 1987, 1989; Hewett et al. 1991; Chaffee et al. 1991; Morris et al. 1991) a pair of high redshift $(z>3)$ quasi-stellar objects (QSOs) were discovered separated by only 8 arcmin. They are $2138-4427(z$ $=3.17)$, and 2139-4434 $(z=3.23)$. Two strong (observed-frame equivalent width [EW] $>30 \AA$ ) absorption systems shortward of the Lyman- $\alpha$ emission were seen in $2138-4427$, one (A) at $z=2.380$ and the other (B) at $z=2.853$. Strong absorption systems $(\mathrm{EW}>10 \AA)$ are seen at both these redshifts in $2139-4434$ as well.

Absorption systems with these equivalent widths are normally interpreted as Lyman- $\alpha$ absorption in galaxy disks on the line of sight (Wolfe et al. 1986), but at these redshifts the absorbers would need a transverse size of $\sim 3$ Mpc (proper distance) to obscure both lines of sight.

The spectra are presented and analyzed in Sec. 2, the interpretation discussed in Sec. 3, and the results summarized in Sec. 3. A Friedmann-Robertson-Walker metric with $q_{0}=0.5$ and $H_{0}=100 \mathrm{hm} \mathrm{s}^{-1} \mathrm{Mpc}^{-1}$ is assumed.

\section{OBSERVATIONS}

$2138-4427$ has a $B_{j}$ magnitude of $18.93 \pm 0.15$, and Equinox 1950.0 coordinates 21:38:44.4-44:27:08. 2139 -4434 is brighter $\left(B_{j}=18.66\right)$ and its 1950.0 coordinates are 21:39:14.7-44:34:01. The original survey observations were made with the Du Pont Telescope of the Las Campanas Observatory (LCO). Higher resolution spectra were subsequently taken of the (A) absorption system at the Anglo-Australian Telescope (AAT).

The LCO observations are described by Morris et al. (1991). They cover the wavelength range 3400-6600 ̊, at $10 \AA$ resolution, and a signal-to-noise ratio (per resolution element) of $\sim 10$. The wavelength region between the Lyman- $\alpha$ emission and the Lyman limit absorbers is shown in Fig. 1. Note that the redshifts of the Lyman-limit systems are $z=2.9$ in $2138-4427$ and $z=3.2$ in 2139-4434; higher than the redshifts of either the (A) or (B) absorption systems.

The AAT observations were made on the nights of 1991 August 6-9, using the RGO spectrograph, a 1200 grooves/mm grating blazed at $4000 \AA$, and a coated Thompson CCD. The dispersion achieved corresponded to $0.7 \AA$ per pixel, and using a slit width of 1.5 arcsec a resolution of $\sim 1.3 \AA$ was obtained. The wavelength coverage was $4000-4620 \AA$. The slit was aligned along the parallactic angle. Eight exposures of $2700 \mathrm{~s}$ were taken on each object. Copper-argon arc exposures were made before and after each individual object exposure.

Bias frames and tungsten flats were taken at the start and end of each night. Night-to-night variations in bias and flatfields were negligible. Standard data reduction and extraction techniques were adopted, using the $\mathrm{IRAF}^{1}$ image

${ }^{1}$ IRAF is distributed by the National Optical Astronomy Observatories, which is operated by the Association of Universities for Research in Astronomy, Inc. (AURA) under cooperative agreement with the National Science Foundation. 


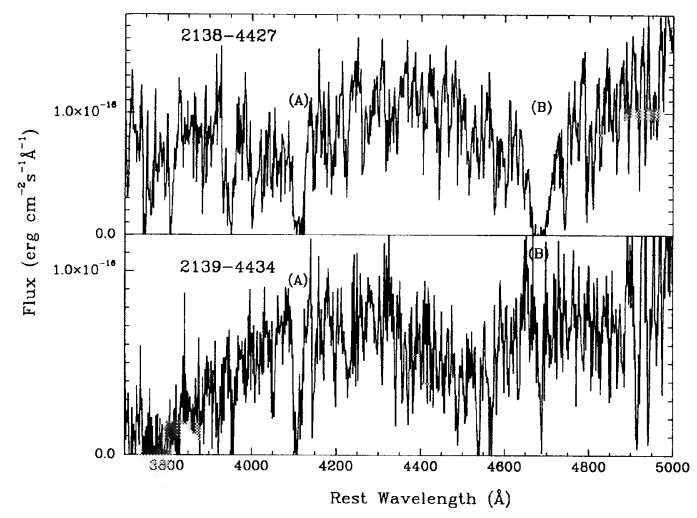

FIG. 1. The Lyman- $\alpha$ forest regions of the LCO spectra. The data are unsmoothed, and plotted in $1.3 \AA$ bins.

reduction system. The copper-argon calibration exposures yielded 20-30 usable lines and a third-order polynomial fit for the wavelength solution gave rms residuals of $\sim 0.04 \AA$. The signal-to-noise ratio in the coadded spectra, Fig. 2, is $\sim 20$ per resolution element.

\section{RESULTS}

The measured parameters of the absorption-line systems are given in Table 1. Equivalent widths were measured interactively by assigning a straight-line continuum and integrating beneath it. The adopted continua and wavelength integration limits are indicated in Fig. 3. The measurements were made only using the LCO spectra, to ensure consistency with the statistical measurements described in Sec. 4.1. Measurements made from the AAT spectra were not significantly different.

An attempt was made to derive central wavelengths and column densities for the absorption systems by fitting Voigt profiles. Given the low signal-to-noise ratios of the LCO spectra and the probable presence of many unresolved blends of Lyman- $\alpha$ forest lines, formal fitting techniques such as $\chi^{2}$ were inappropriate and a more subjective process was used. Synthetic Voigt profiles were generated, as-

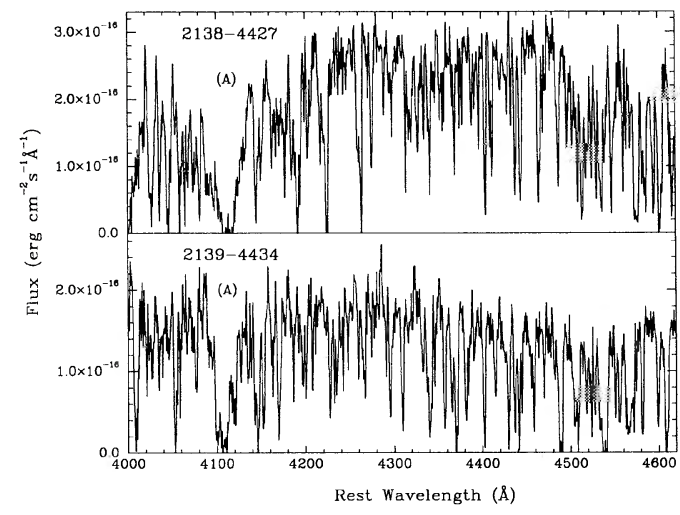

FIG. 2. The AAT spectra. The data are unsmoothed, and plotted in $0.5 \AA$ bins.
TABLE 1. Absorption system parameters.

\begin{tabular}{lcccc}
\hline \hline QSO & System & EW $(\AA)$ & Center $(\AA)$ & Column Density $\left(\mathrm{cm}^{-2}\right)$ \\
\hline 2138 & (A) & 30 & 4112.7 & $2.5 \times 10^{19}$ \\
2139 & (A) & 21 & 4108.7 & $7.0 \times 10^{18}$ \\
2138 & (B) & $>60$ & 4682 & $1.2 \times 10^{20}$ \\
2139 & (B) & 11 & 4686 & $1.2 \times 10^{18}$ \\
\hline \hline
\end{tabular}

suming a constant continuum level. They were visually compared to the observed absorption profiles, and the column densities, central wavelengths, and temperatures assumed were varied until a good visual match was obtained.

The adopted matches are shown in Fig. 4. The single Voigt profile is an acceptable fit to both systems in 2138 -4427 , and to system (B) in 2139-4434. For system (A) in 2139-4434, however, no single absorber could match the observed profile. Instead, an attempt was made to fit the central dip only. This narrower Voigt profile is shown.

The results were found to be insensitive to the assumed temperature-the linewidths are dominated by atomic processes (a temperature of $30000 \mathrm{~K}$ was used). A factor of two increase or decrease in the assumed column densities resulted in unacceptably bad matches between the models and data.

The presence of other transitions arising in the absorption systems was investigated by producing velocity plots of the predicted locations of the strongest absorption lines listed by Morton et al. (1988). The LCO data (Fig. 5) shows possible Lyman- $\beta$ and $-\gamma$ from system (B) in 2138 -4427 but nothing convincing in $2139-4434$. The AAT data (Fig. 6) shows probable low ionization metal lines from system (A) in $2138-4427$, but nothing in 2139 -4434 .

Given the low signal-to-noise ratio and limited resolution of the data, we do not attempt to derive a metallicity for system (A) in 2138-4427. However, we note that the $\sim 3 \AA$ observed-frame equivalent width of $\mathrm{O}_{\mathrm{I}}(1302 \AA)$ is typical of absorption systems with approximately solar metallicity (Wolfe et al. 1993; Lu et al. 1993).
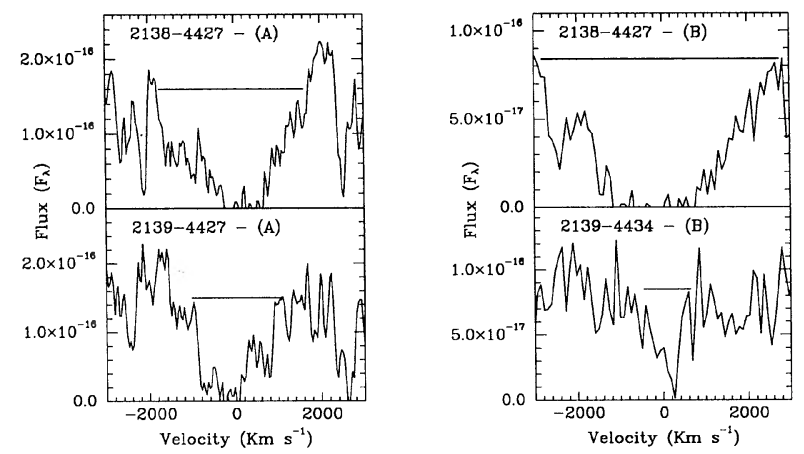

FIG. 3. The adopted continuum and integration limits for computing equivalent widths. The horizontal lines show the adopted continuum, and the integration limits are the ends of the lines. To compute velocities, a redshift of 2.380 was used for system (A), and one of 2.853 for system (B). LCO spectra are shown for system (B) and AAT spectra are shown for system (A). 

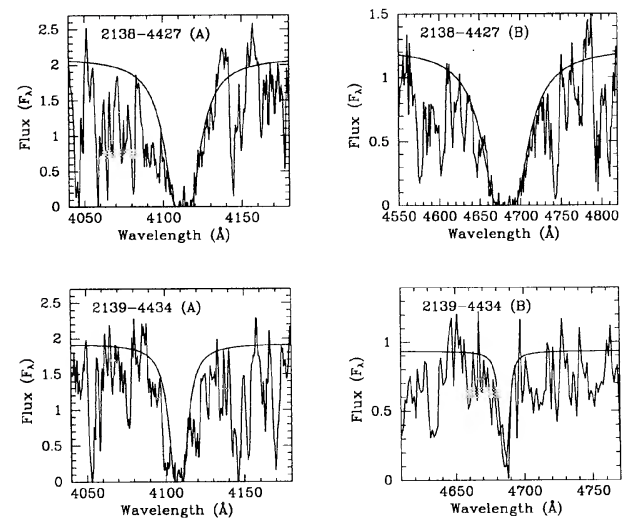

FIG. 4. Voigt profile fits to the spectra. A horizontal continuum has been used. AAT spectra are shown for system (A) and LCO spectra for system (B). The fluxes are in units of $10^{-16} \mathrm{erg} \mathrm{cm}^{-2} \mathrm{~s}^{-1} \AA^{-1}$.

\section{DISCUSSION}

Three possible explanations for the presence of the absorbers are: (a) chance coincidences, (b) high-redshift galaxy superclusters, and (c) individual Mpc scale gas clouds.

\subsection{Coincidence Probabilities}

To determine the average line-of-sight density of Lyman- $\alpha$ absorbers with large equivalent widths, all 34 LBQS QSOs with redshifts above 2.7 which are not broad absorption line QSOs were examined. The equivalent widths of all absorption lines visually estimated to have observed-frame equivalent-widths greater than $5 \AA$ were measured using the interactive technique described in Sec. 3.

A total pathlength of $45000 \AA$ between the Lyman- $\alpha$ emission of the QSOs and either the Lyman limit or the UV atmospheric cutoff was surveyed. Obvious blends were not counted as single lines, but all ambiguous cases were conservatively counted as single systems. 78 absorbers with observed-frame equivalent widths greater than $10 \AA$ were seen, 15 with equivalent widths greater than $20 \AA$, and 3 with equivalent widths greater than $30 \AA$.

The wavelength differences between the lines in the two lines of sight are $<5 \AA$. Taking the null hypothesis that absorbers in the two lines of sight are uncorrelated, the
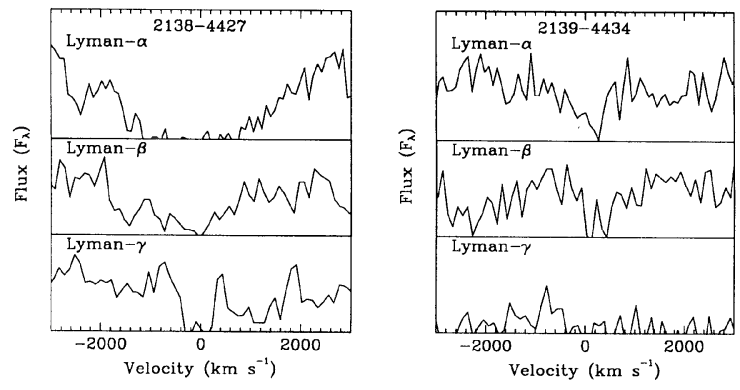

FIG. 5. The positions of strong hydrogen absorption lines at the redshift of the system (B) Lyman- $\alpha$ absorbers. LCO spectra are shown, and a redshift of 2.853 assumed.
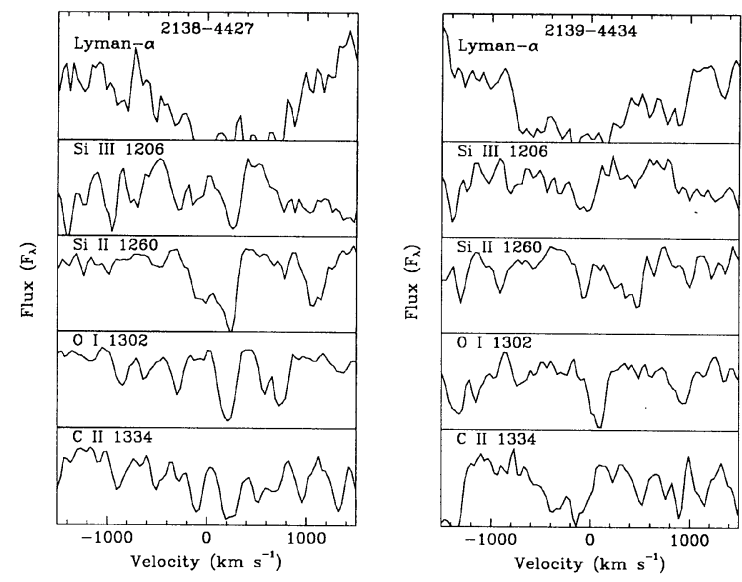

FIG. 6. The positions of strong metal-line absorbers at the redshift of the system (A) Lyman- $\alpha$ absorbers. AAT spectra are shown, and a redshift of 2.380 assumed.

probability that a $>10 \AA$ absorber [such as (B) in 2139 -4434] would lie within $5 \AA$ of a given wavelength is 1.7\%. The probability for a $>20 \AA$ absorber [such as (A) in $2139-4434$ ] is $0.3 \%$.

To compute the joint probability of these coincidences, two additional factors must be taken into account. Firstly, attention was drawn to this QSO pair because of system (A), system (B) being discovered later on a detailed examination of the spectra. Thus a match such as (B) would not alone have been noticed. Secondly, in all the LBQS high-redshift QSO pairs with separations of 10 arcmin or less, one other strong absorber (with an equivalent width $>20 \AA$ ) was observed, and this does not have any coincident absorption in the other line of sight. Thus the relevant joint probability is that of observing one wavelength coincidence with an equivalent-width $>20 \AA$ system and one with an equivalent-width $>10 \AA$ system with three trials. This probability is $3 \times 10^{-4}$.

This probability analysis holds even if the absorbers are blends of low column density lines and not single absorbing clouds. As the wavelength densities of absorbers were measured from data with comparable noise and resolution to the LCO spectra of $2138-4427$ and $2139-4434$, they should all be contaminated by line blends to the same extent. Thus even if blending is important, the probability of having two blends this strong this close in wavelength is still $3 \times 10^{-4}$.

\subsection{Superclusters}

One possible explanation of the observed redshift coincidences is that the two lines of sight pass through two different galaxy disks in each of two superclusters. The comoving separation of the two lines of sight is $\sim 10 h^{-1}$ Mpc for $q_{0}=0.1$, or $\sim 6 h^{-1} \mathrm{Mpc}$ for $q_{0}=0.5$, assuming $H_{0}=100 \mathrm{~h} \mathrm{~km} \mathrm{~s}^{-1} \mathrm{Mpc}^{-1}$ and a Friedmann-RobertsonWalker metric, so a supercluster scale structure is indicated.

The galaxy density of the cluster can be gauged from the probabilities in Sec. 4.1. To raise the $a$ posteriori probability 
of the matches to $5 \%$, an overdensity of absorbers (compared to the field) of a factor of 30 is required.

Alternatively, consider a spherical cluster of radius 10 Mpc (comoving), filled uniformly with identical galaxies which absorb out to a radius $r$ kpcs. If $r=10 \mathrm{kpc}$, about five galaxies would be needed per comoving cubic Mpc to yield an a posteriori probability of the wavelength matches of $5 \%$. For $r=50 \mathrm{kpc}$, a comoving density of 0.2 galaxies per cubic Mpc is required. The supercluster would include $10^{4}(r=10 \mathrm{kpc})$ or $5 \times 10^{2}(r=50 \mathrm{kpc})$ galaxies.

These inferred supercluster overdensities are roughly a factor of ten greater than the real space galaxy overdensities of present day superclusters. However, present day "Great Wall" type structures can show comparable overdensities in velocity space when observed face on, due to the infall of individual galaxies. A face-on planar geometry for the absorbing systems is also suggested by remarkably small velocity differences between the absorbers, as if they are moving with the Hubble flow, their velocity differences correspond to proper distance separations along the line of sight of $\sim 3 \mathrm{Mpc}$, comparable to their transverse separation.

Thus "Great Wall" galaxy superclusters may explain the matches. However most structure formation theories, especially "bottom-up" scenarios such as Cold Dark Matter (e.g., Davis et al. 1985) predict that superclusters formed at redshifts below 1 .

\subsection{Giant Gas Clouds}

In a model in which the absorption is due to a single Mpc scale gas cloud, we can estimate the cloud mass. For a disk geometry, oriented perpendicular to the line of sight, with a diameter just sufficient to obscure both lines of sight (3 $\mathrm{Mpc}$ proper distance), and a mean neutral hydrogen column density of $10^{19} \mathrm{~cm}^{-2}$, the total mass is $10^{11} \mathscr{M}_{\odot}$. If system (B) in $2138-4427$ is more typical of the column density of the cloud, or if the cloud is larger than $3 \mathrm{Mpc}$, the mass increases correspondingly.

Uson et al. (1991) detected $21 \mathrm{~cm}$ absorption and emission at redshift 3.4 separated by 33 arcmin. Their column densities depend on the unknown temperature of the gas, but, given their assumed temperature, are an order of magnitude greater than those measured here. Our assumed size is a lower limit, and does not preclude our systems also extending over 30 arcmin. They interpret their findings as a Zel'dovich pancake, and ours could be interpreted similarly, though the presence of metals in at least one of the systems argues for some stellar processing.

\section{CONCLUSIONS}

The $a$ posteriori probabilities of these redshift coincidences are low, but given the inherent problems with such statistics, no single pair of objects can be compelling. However, these absorption systems join a small but growing family of observations of possible large scale structures at high redshifts. Sargent (1987) reports high column density absorption systems at identical redshifts in the QSO pair Q2343 + 1229 and Q2343+1225 separated by 5.7 arcmin. Robertson (1987), Cristiani et al. (1987), Sargent \& Steidel (1987), and Jakobsen \& Perryman (1992) discuss the QSO pair Tololo 1037-27/1038-27, separated by 18 arcmin, in which possible metal-line absorption system wavelength coincidences are seen. Uson et al. detected $21 \mathrm{~cm}$ absorption and emission at redshift 3.4 separated by 33 arcmin. On a larger scale, a substantial overdensity of metal-line absorbers is seen in the spectrum of QSO PKS 0237-233 (Heisler et al. 1989; Foltz et al. 1993).

These observations all hint at the presence of large dense structures in the early universe, which would impose a considerable challenge to our understanding of the early universe.

We wish to thank Jill Bechtold, Craig Foltz, and Simon White for helpful discussions, and Shoba Veeraraghavan and Steve Warren for pointing out an error in a previous draft. P.J.F. is supported by a SERC/NATO advanced fellowship. Computing support was provided in part by the Starlink Project, which is funded by the UK SERC. We also thank the staff at the AAT for their invaluable assistance.

\section{REFERENCES}

Chaffee, F. H., Foltz, C. B., Hewett, P. C., Francis, P. J., Weymann, S. L., Morris, S. L., Anderson, S. F., \& MacAlpine, G. M. 1991, AJ, 102, 461 Cristiani, S., Danziger, I. J., \& Shaver, P. A. 1987, MNRAS, 227, 639 Davis, M., Efstathiou, G., Frenk, C. S., \& White, S. D. M. 1985, ApJ, 292, 371

Foltz, C. B., Chaffee, F. H., Hewett, P. C., MacAlpine, G. M., Turnshek, D. A., Weymann, R. J., \& Anderson, S. F. 1987, AJ, 94, 1423

Foltz, C. B., Chaffee, F. H., Hewett, P. C., Weymann, R. J., Anderson, S. F., \& MacAlpine, G. M. 1989, AJ, 98, 1959

Foltz, C. B., Hewett, P. C., Chaffee, F. H., \& Hogan, C. J. 1993, AJ, 105, 22

Heisler, J., Hogan, C. J., \& White, S. D. M. 1989, ApJ, 347, 52

Hewett, P. C., Foltz, C. B., Chaffee, F. H., Francis, P. J., Weymann, R. J., Morris, S. L., Anderson, S. F., \& MacAlpine, G. M. 1991, AJ, 101, 1121

Jakobson, P., \& Perryman, M. A. C. 1992, ApJ, 392, 432

Lu, L., Wolfe, A. M., Turnshek, D. A., \& Lanzetta, K. M. 1993, ApJS, 84,1
Morris, S. L., Weymann, R. J., Anderson, S. F., Hewett, P. C., Foltz, C. B., Chaffee, F. H., Francis, P. J., \& MacAlpine, G. M. 1991, AJ, 102, 1627

Morton, D., York, D., \& Jenkins, E. 1988, ApJS, 68, 449

Robertson, J. G. 1987, MNRAS, 227, 653

Sargent, W. L. W. 1987, in QSO Absorption Lines, Probing the Universe, edited by J. C. Blades, D. Turnshek, and C. A. Norman (Cambridge University Press, Cambridge), p. 8

Sargent, W. L. W., \& Steidel, C. C. 1987, ApJ, 322, 142

Uson, J. M., Bagri, D. S., \& Cornwell, T. J. 1991, Phys. Rev. Lett., 67, 3328

Wolfe, A. M., Turnshek, D. A., Smith, H. E., \& Cohen, R. D. 1986, ApJS, 61, 249

Wolfe, A. M., Turnshek, D. A., Lanzetta, K. M., \& Lu, L. 1993, ApJ (in press) 